\title{
Whole Transcriptome Analysis of Hypertension Induced Cardiac Injury Using Deep Sequencing
}

\author{
Tao-Tao Li Xiao-Yan Li Li-Xin Jia Jing Zhang Wen-Mei Zhang Yu-Lin Li \\ Yong-Fen Qi Jie Du \\ Beijing Anzhen Hospital, Capital Medical University; The Key Laboratory of Remodeling-Related \\ Cardiovascular Diseases, Ministry of Education; Beijing Collaborative Innovation Center for \\ Cardiovascular Disorders; Beijing Institute of Heart, Lung \& Blood Vessel Disease, Beijing, China
}

\section{Key Words}

Hypertension • Cardiac injury • Transcriptome analysis • RNA-Seq • Ubiquitin C

\begin{abstract}
Background/Aims: Hypertension plays a critical role in the cardiac inflammation and injury. However, the mechanism of how hypertension causes the cardiac injury at a molecular level remains to be elucidated. Methods: RNA-Seq has been demonstrated to be an effective approach for transcriptome analysis, which is essential to reveal the molecular constituents of cells and tissues. In this study, we investigated the global molecular events associated with the mechanism of hypertension induced cardiac injury using RNA-Seq analysis. Results: Our results showed that totally 1,801 genes with different expression variations were identified after Ang II infusion at 1, 3 and 7 days. Go analysis showed that the top 5 high enrichment Go terms were response to stress, response to wounding, cellular component organization, cell activation and defense response. KEGG pathway analysis revealed the top 5 significantly overrepresented pathways were associated with ECM-receptor interaction, focal adhesion, protein digestion and absorption, phagosome and asthma. Moreover, protein-protein interaction network analysis indicated that ubiquitin $C$ may play a key role in the processes of hypertension-induced cardiac injury. Conclusion: Our study provides a comprehensive understanding of the transcriptome events in hypertension-induced cardiac pathology.
\end{abstract}

\section{Introduction}

Hypertension induces damage to heart, kidney, brain, and eye [1, 2]. As a central effector of hypertension, dysregulated angiotensin II (Ang II) triggers a set of inflammatory responses $[3,4]$ and cardiomyocyte hypertrophy $[5,6]$ leading to organ damage. We and others have 
demonstrated that the inflammatory responses in heart promote a forward feedback infiltration of leukocytes, differentiation of monocytes, and formation of myofibroblasts. Myofibroblasts, which is the dominant source of extracellular matrix production, results in deposition of collagen and cardiac injury [3, 7-9]. However, the mechanism of how hypertension causes the cardiac injury at a molecular level remains to be elucidated.

Transcriptome analysis provides global, integrated and specified information of a tissue at a specific moment [10]. Various technologies have been developed for transcriptome analysis. Although microarray has been widely used, this technology has several defects and limitations. For example, the background level is high because of cross-hybridization and the dynamic range for detection is limited because of background and saturated signals [11]. Recent advances in high-throughput sequencing technologies are enabling a new way to study transcriptomes: massively parallel sequencing of short reads derived from mRNAs (RNA-Seq) $[12,13]$. One advantage of this method is that RNA-Seq is not limited to detecting existing genomic sequence compared with hybridization-based microarray [12]. A second advantage of RNA-Seq relative to microarray is that RNA-Seq has low background signal because DNA sequences can been unambiguously mapped to unique regions of the genome [12]. Because there are no cloning steps, and with the Helicos technology there is no amplification step, RNA-Seq requires less RNA sample [12]. Besides, RNA-Seq has been shown to be highly accurate for quantifying expression levels $[14,15]$. The results of RNASeq also show high levels of reproducibility, for both technical and biological replicates [16]. Recently, RNA-Seq has been demonstrated to be an effective approach for investigating the pathogenesis of complex heart diseases, including cardiac hypertrophy and heart failure [17-20].

In this study, we identified 1,801 differently expressed genes at 1, 3 and 7 days after Ang II infusion by using RNA-Seq analysis. Go and KEGG pathway analysis showed that the high enrichment Go terms were mainly response to stress, response to wounding, cellular component organization, cell activation and defense response; the key pathways included ECM-receptor interaction, focal adhesion, protein digestion and absorption, phagosome and asthma. Besides, protein-protein interaction network analysis indicated that ubiquitin $\mathrm{C}$ may play a key role in the processes of hypertension-induced cardiac injury.

\section{Materials and Methods}

\section{Animal model and treatments}

To induce hypertension, osmotic minipumps (Alzet Model 1007D, Alzet Model 1003D, DURECT, Cupertino, CA) filled with Ang II or acetic acid saline were placed subcutaneously in the intrascapular area to deliver Ang II at a concentration of $1500 \mathrm{ng} / \mathrm{kg}$ per minute as described previously [3]. Systolic blood pressure was measured at the indicated day using a computerized mouse tail cuff system (BP98A, Softron, Tokyo, Japan) as described elsewhere [21]. The Guide for the Care and Use of Laboratory Animals (National Institutes of Health Publication No. 85-23, 2006) was followed, and the study was approved by the Animal Care and Use Committee of Capital Medical University.

Flow cytometry

Cardiac inflammatory cell infiltrates were quantified by flow cytometry as described previously [9]. Briefly, mouse hearts were minced and digested with $200 \mathrm{U} / \mathrm{mL}$ collagenase II and $2.4 \mathrm{U} / \mathrm{mL}$ dispase II in PBS at $37^{\circ} \mathrm{C}$ for 30 minutes. Cell suspensions were filtered and collected at $300 \times \mathrm{g}$ for 10 minutes. Flow cytometry was carried out using the following antibodies: PE anti-mouse F4/80 (Biolegend, San Diego, CA), PE-cy7 anti-mouse Ly6G, PerCP-Cy5.5 anti-mouse CD45.2, and 488 anti-mouse CD11b (all from BD Biosciences, San Jose, CA). Stained cells were analyzed by flow cytometry, and data were collected using Summit 5.2 software (Beckman Coulter, Miami, FL).

Histology and immunohistochemical analysis

Mouse heart tissue was fixed in $4 \%$ paraformaldehyde, embedded in paraffin, and sectioned. Heart sections $(5 \mu \mathrm{m})$ were stained with Masson's trichrome reagents [3,22]. Images were captured by the Nikon 


\section{Cellular Physiology Cell Physiol Biochem 2016;38:670-682

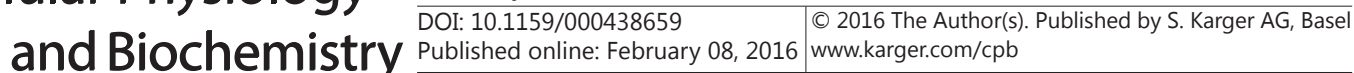 \\ Li et al.: Transcriptome Analysis in Cardic Injury}

microscope eclipse 90i (Nikon, Tokyo, Japan). The percent of fibrosis (green staining) to total tissue was calculated by a person blinded to treatment with the use of NIS-Elements BR 3.1 software (Nikon).

RNA extraction, whole-transcriptome sequencing

Total RNA was extracted with TRizol (Invitrogen, Carlsbad, CA) according to the manufacturer's protocol, followed by DNase I treatment to eliminate DNA contamination. Equal quantities of RNA from 5 samples in one group were mixed. The mRNA was enriched and fragmented into short fragments (approximately $200 \mathrm{bp}$ ). The double-stranded cDNA was synthesized and purified before sequencing adaptors were ligated to the fragments. For quality control, an Agilent 2100 Bioanalyzer and ABI StepOnePlus Real-Time PCR System were used to qualify and quantify the sample library. The library products were finally sequenced via Illumina HiSeq ${ }^{\mathrm{TM}} 2000$. No less than $11 \mathrm{M}$ total reads were obtained from each sample.

Validation of RNA-Seq data by qPCR analysis

Equal amounts of RNA ( $2 \mu \mathrm{g}$ ) were added to reverse transcriptase reaction mix with oligo-dT primers (Promega, Southampton, UK). SYBR Premix Ex Taq (TaKaRa, Shiga, Japan) was used to perform quantitative real-time PCRs with IQ5 Multicolor Real-time PCR Detection System (Bio-Rad, Hercules, CA). The following primers were used: Igfbp5 (5'-CTC ATG ATT CCA AAC GTG TGTTG-3' forward; 5'-TGG GCT ATG CAC TTG ATGCA-3' reverse), Slc38a3 (5'-CCA TTA CGC TTC AGA ACA TTGG-3' forward; 5'-GAG GCA ATT CAG ACT TGA TGA TGTA-3' reverse), Rnf207 (5'-CGA GGA CAC ACC CTT CAC AGA-3' forward; 5'-GCA GAC CCC GAT AGG AATCC-3' reverse), Vim (5'-GAG AGA GGA AGC CGA AAGCA-3' forward; 5'-GCC AGA GAA GCA TTG TCA ACATC-3' reverse), GAPDH (5'-CAT GGC CTT CCG TGT TCCTA-3' forward; 5'-GCG GCA CGT CAG ATCCA-3' reverse).

Analysis of RNA-Seq data

As there are some adaptor sequences and/or low quality reads present in the raw reads, data filtering is carried out to obtain high quality reads as the clean reads (clean data). Clean reads were mapped to reference sequences and/or reference gene set using SOAP aligner/SOAP2 [1] [23]. No more than 2 mismatches were allowed in the alignment. Then, a comprehensive bioinformatics analysis was used to enrich the dataset for genes that were most likely to be associated with the development of Ang II-induced acute cardiac injury, including clustering analysis, Gene ontology (GO) analysis, pathway analysis, and dynamic gene network analysis.

\section{Statistical analysis}

Data were expressed as the means \pm standard error of the mean (SEM). Differences between groups were tested for statistical significance using Student's t-tests or one-way analysis of variance (ANOVA) followed by Newman-Keuls multiple comparison tests using Graph Pad Prism 5.0. P 0.05 denoted the statistically significant difference.

\section{Results}

Ang II stimulated cardiac inflammation and fibrosis in mice

Hypertensive mouse model was successfully built by Ang II infusion. The blood pressure was markedly increased in a time-dependent manner (at days 1, 3 and 7) (Fig. 1A). Inflammatory cell infiltration into the heart was examined by flow cytometry. As shown in Fig. 1B, Ang II infusion significantly increased the infiltration of CD45 leukocytes, $\mathrm{CD}_{4} 5^{+} \mathrm{CD} 11 \mathrm{~b}^{+} \mathrm{Ly}_{6 \mathrm{G}^{+} \mathrm{F} 4 / 80}$ neutrophils, $\mathrm{CD} 45^{+} \mathrm{CD} 11 \mathrm{~b}^{+} \mathrm{F} 4 / 80^{+}$macrophages into the heart at days 1, 3 and 7. Pathological examination indicated Ang II increased extracellular matrix deposition, determined by Masson's trichrome staining (Fig. 1C).

\section{Identification of differentially expressed genes (DEGs)}

To investigate the DEGs in hypertension-induced cardiac injury at early stage, heart tissues were taken from the mice at days 1, 3, and 7 after Ang II infusion. Then a time series RNA-Seq were performed. We obtained more than 11 million total sequence reads per sample. As shown in Fig. 2A, after filtering adaptor sequence and low quality reads, the presents of clean reads in raw reads were all more than $98.5 \%$ in four samples. 


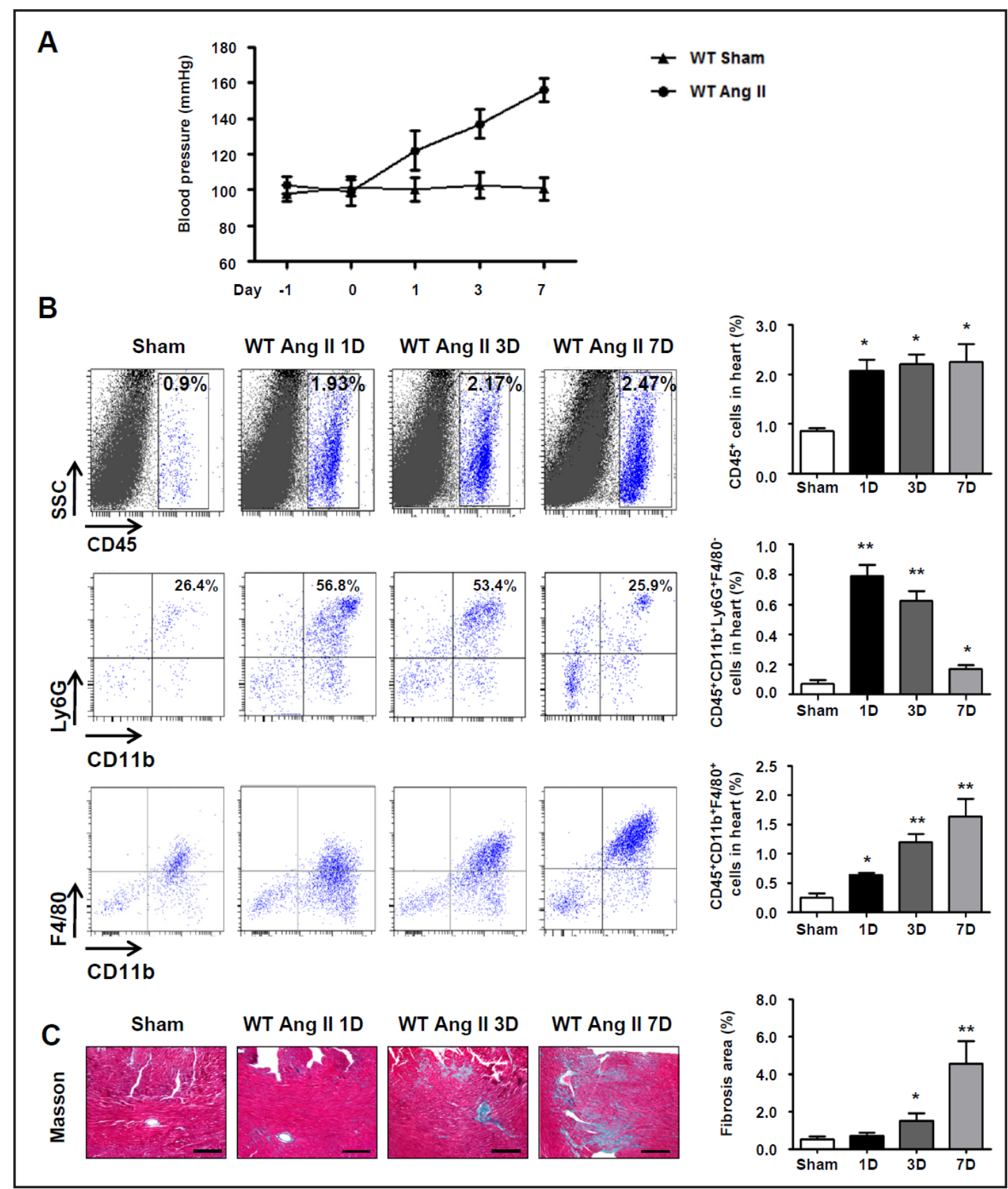

Fig. 1. Ang II stimulated cardiac inflammation and fibrosis in mice. (A) Systolic blood pressure was measured by the tail-cuff method at different time points after Ang II infusion. ( $n=4$ in each group). (B) Flow cytometry analysis of $\mathrm{CD} 45^{+}$leucocytes, $\mathrm{CD} 45^{+} \mathrm{CD} 11 \mathrm{~b}^{+} \mathrm{Ly} 6 \mathrm{G}^{+} \mathrm{F} 4 / 80^{-}$neutrophils and $\mathrm{CD} 45^{+} \mathrm{CD} 11 \mathrm{~b}^{+} \mathrm{F} 4 / 80^{+}$ macrophages were performed at different time points after Ang II infusion. Bar graph shows the percentage of cells in the heart ( $\mathrm{n}=4$ in each group). (C) Masson staining of fibrosis and area quantitation in WT mouse hearts at different time points after Ang II infusion. (scale bars, $100 \mu \mathrm{m} ; \mathrm{n}=4$ in each group). ${ }^{*} P<0.05$ vs. Sham. ${ }^{* *} P<0.01$ vs. Sham.

To judge the significance of gene expression difference, we use "FDR $\leq 0.001$ and the absolute value of $\log 2$ Ratio $\geq 1$ " as the threshold [24]. When compared with genes in the saline-treated group (Sham), 195 genes significantly increased and 308 genes decreased in the $1 \mathrm{~d}$ group; 1023 genes markedly increased and 558 genes decreased in the $3 \mathrm{~d}$ group, and 397 genes increased and 40 genes decreased in the $7 \mathrm{~d}$ group (Fig. 2B). Moreover, we KARGER 
Fig. 2. The distributions of upand down-regulated genes at different time points after Ang II infusion. (A) Composition of raw reads at different time points after Ang II infusion. (B) Bar gragh shows the number of up- and down-regulated genes at day 1 , 3, 7 compared with Sham. (FDR $\leq 0.001$ and $\log 2$ $\geq 1$ ).

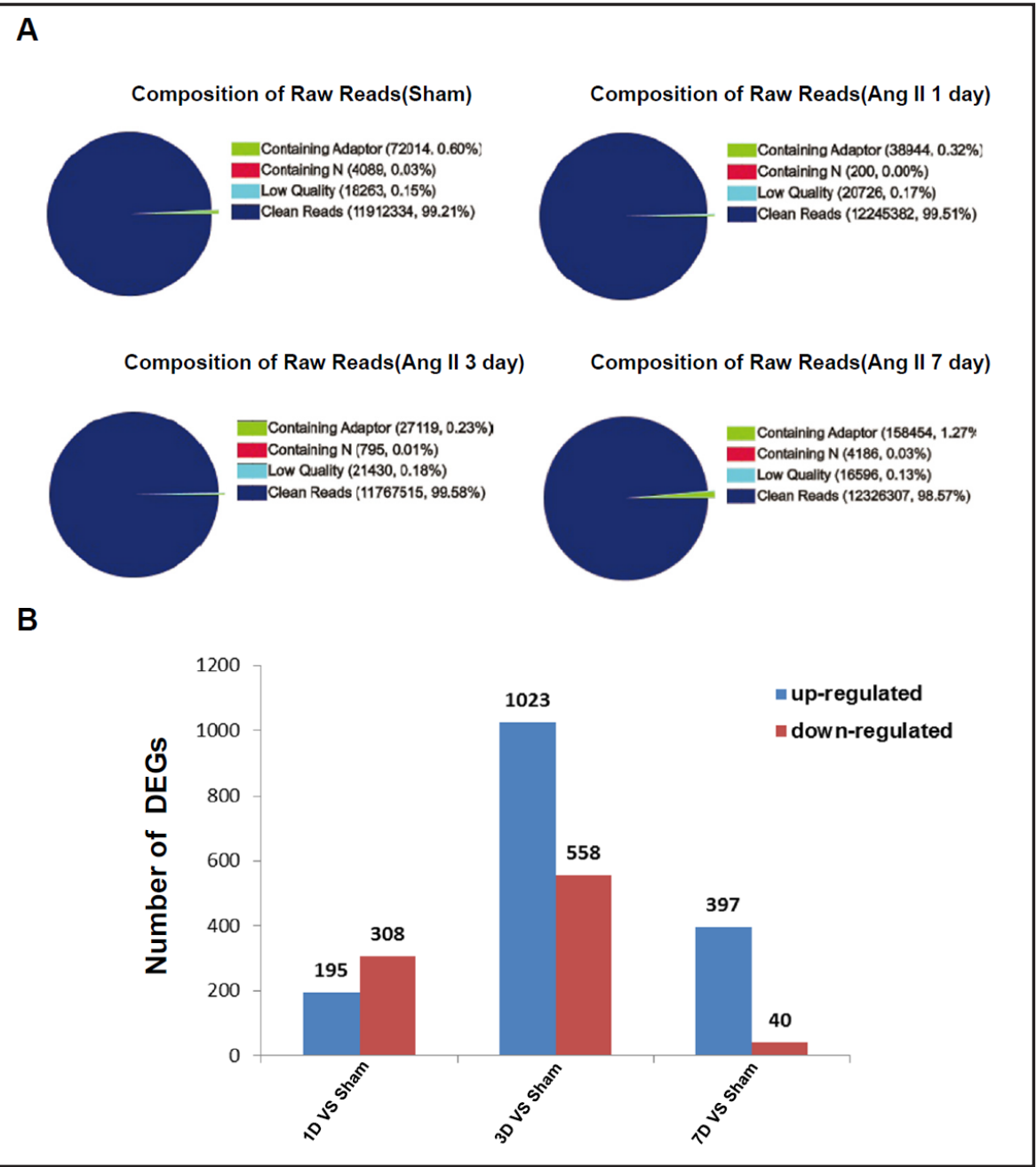

found that total 1,801 genes were shown to be significantly expressed in Ang II-infused mice at least at one time point when compared to Sham. About 58 genes were shown to be significantly expressed in Ang II-infused mice at all three time points when compared to Sham (FDR $\leq 0.001$ and $\log 2 \geq 1$ ).

\section{Gene Ontology (GO) Analysis}

To examine the characteristics of differentially expressed genes, functional classification of up- and down-regulated genes was performed using the gene ontology (GO) tool. In Fig. 3 , we showed the most statistically significantly overrepresented Go terms of biological process. The top 5 high enrichment Go terms were respectively response to endogenous stimulus, response to hormone stimulus, response to stress, response to wounding and response to chemical stimulus at day 1 after Ang II infusion; response to stress, response to wounding, cellular component organization, cell activation and defense response at day 3; immune system process, extracellular structure organization, anatomical structure development, extracellular matrix organization and response to wounding at day 7 . The results indicated that these GO terms played critical roles in the pathobiology of Ang IIinduced early cardiac injury and fibrosis.

\section{KEGG pathway enrichment analysis of DEGs}

To further understand genes biological functions, we performed the KEGG pathway enrichment analysis [25]. The top 5 significantly overrepresented pathways were respectively associated with rheumatoid arthritis, ribosome, cytokine-cytokine receptor interaction, 
Fig. 3. Gene Ontology (GO) analysis. The significant Go category for differentially expressed genes at day 1(A), 3(B), 7(C) compared with Sham. LgP is the logarithm of $P$-value.

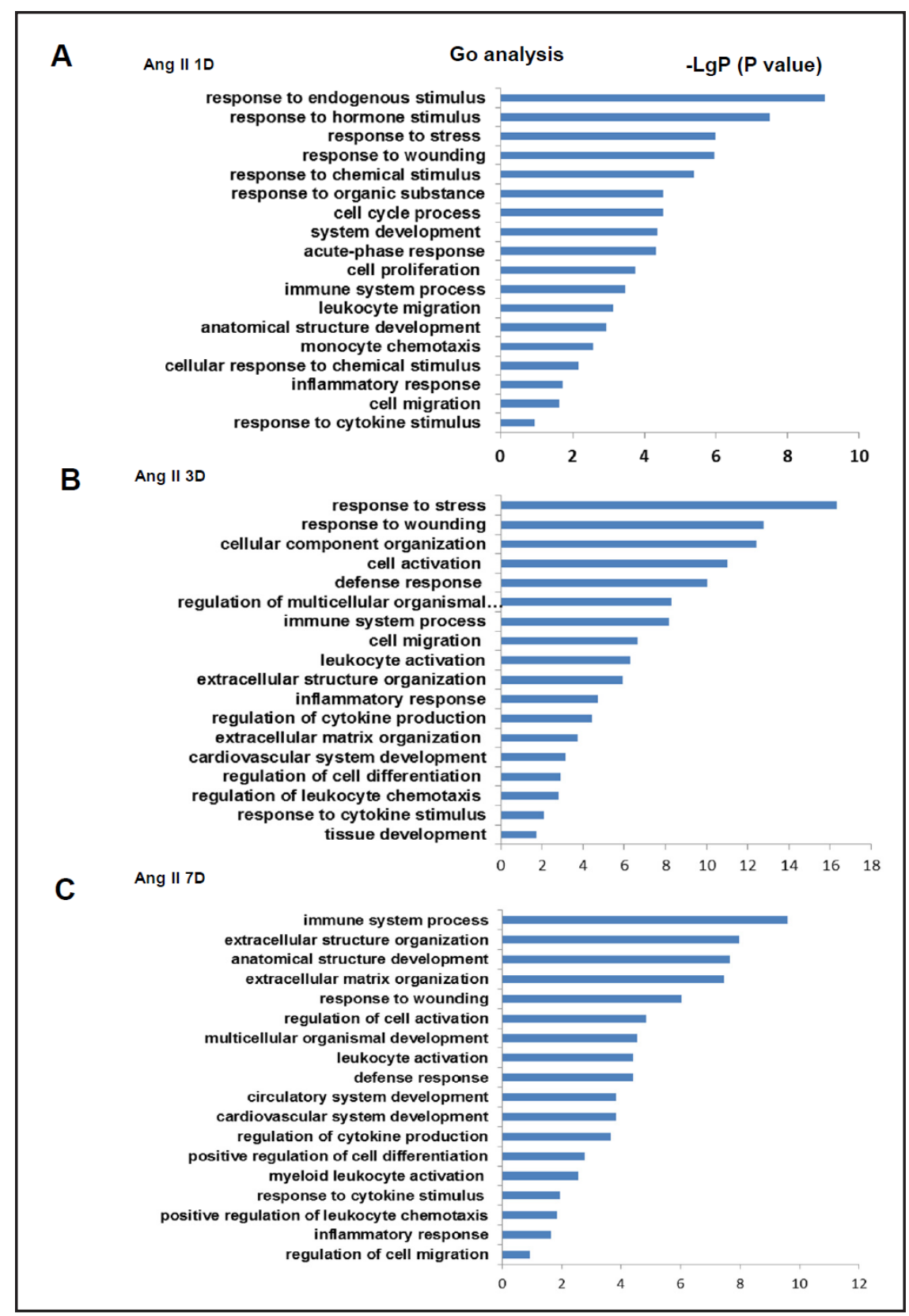

chemokine signaling pathway and asthma at day 1 after Ang II infusion; ECM-receptor interaction, protein digestion and absorption, focal adhesion, phagosome and osteoclast differentiation at day 3; ECM-receptor interaction, focal adhesion, protein digestion and absorption, asthma and phagosome at day 7 (Fig. 4). These pathways at the early phase of Ang II infusion could be considered to be important for Ang II-induced cardiac inflammation and fibrosis.

Cluster analysis of significant differential genes in Ang II-treated hearts

Genes with similar expression patterns usually mean functional correlation. We perform cluster analysis of gene expression patterns with cluster software [26] and Java Tree view software [27]. We do cluster for 2 types as following: For samples' gene expression, log10 (RPKM) will be clustered, and the color is more close to red, the more highly this gene expresses (Fig. 5A). In Fig. 5B, each column represents an experimental condition (e.g. exp1VS-exp2), each row represents a gene. Expression differences are shown in different colors. 
Fig. 4. KEGG pathway enrichment analysis. The significant pathway for differentially expressed genes at day $1(\mathrm{~A}), 3$ (B), 7(C) compared with Sham. LgP is the logarithm of $P$-value.

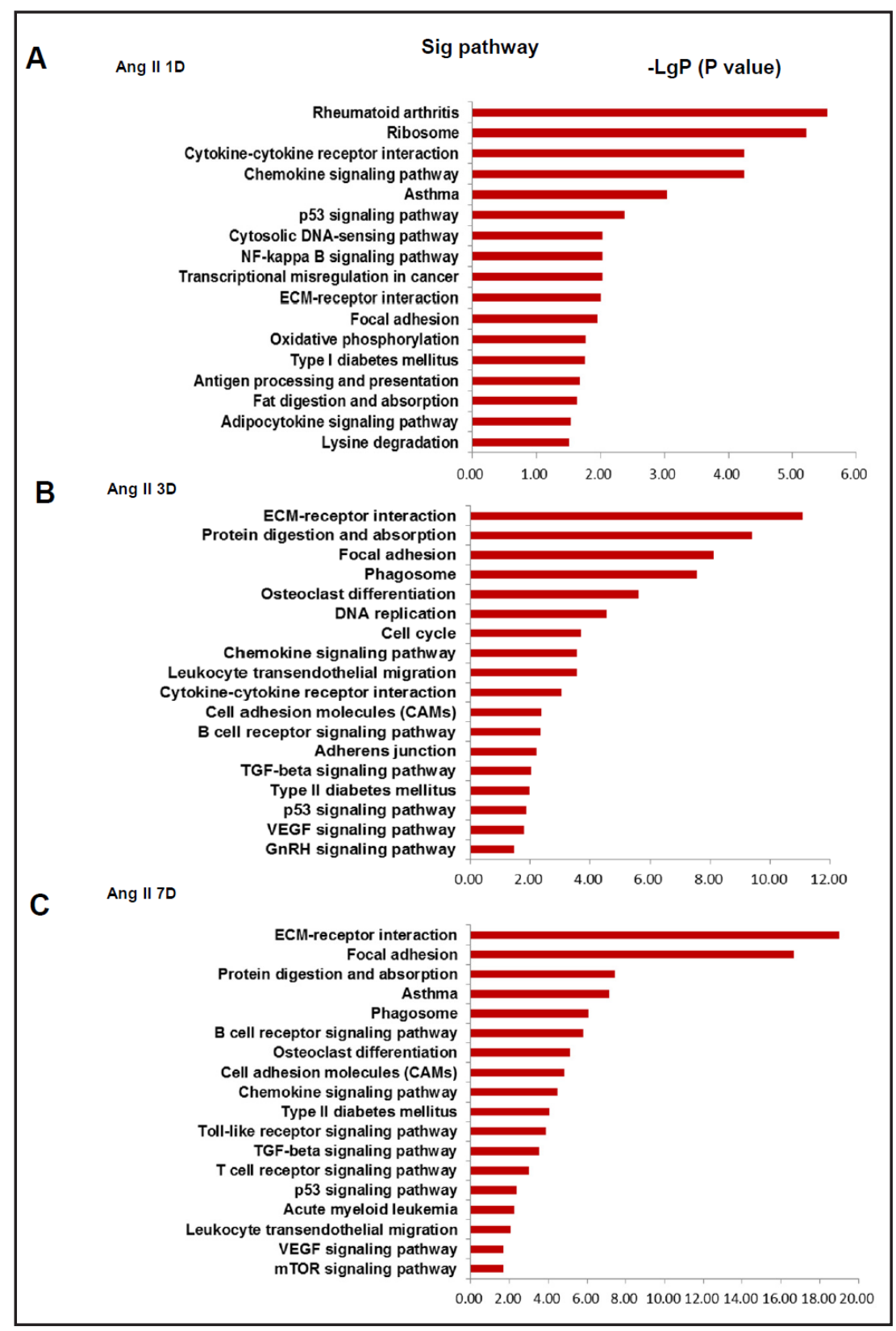

Red means up regulation and blue means down regulation. After cluster analysis, a total 1801 genes were classified into 16 profiles based on expression patterns, and each cluster has similar expression patterns after Ang II infusion. Among these profiles, we identified top 4 profiles of gene expression that contained a total of 1262 genes (Fig. 5C). Thus, these significant profiles were considered to be the potential main expression profiles in our experiment. To confirm the results from RNA-Seq, qPCR was performed. As shown in Fig. $5 \mathrm{D}$, the relative expression levels of 4 differentially expressed genes from the top 4 profiles, including Igfbp5, Slc38a3, Rnf207, Vim, were good consistent between the two experiments.

\section{Protein-protein interaction network analysis}

Different proteins often form protein complex through complicated interactions to perform their biological functions. To further determine which protein or proteins may play key roles in the early stage of acute cardiac injury induced by Ang II infusion, we performed protein-protein interaction network analysis integrating several famous interaction network 


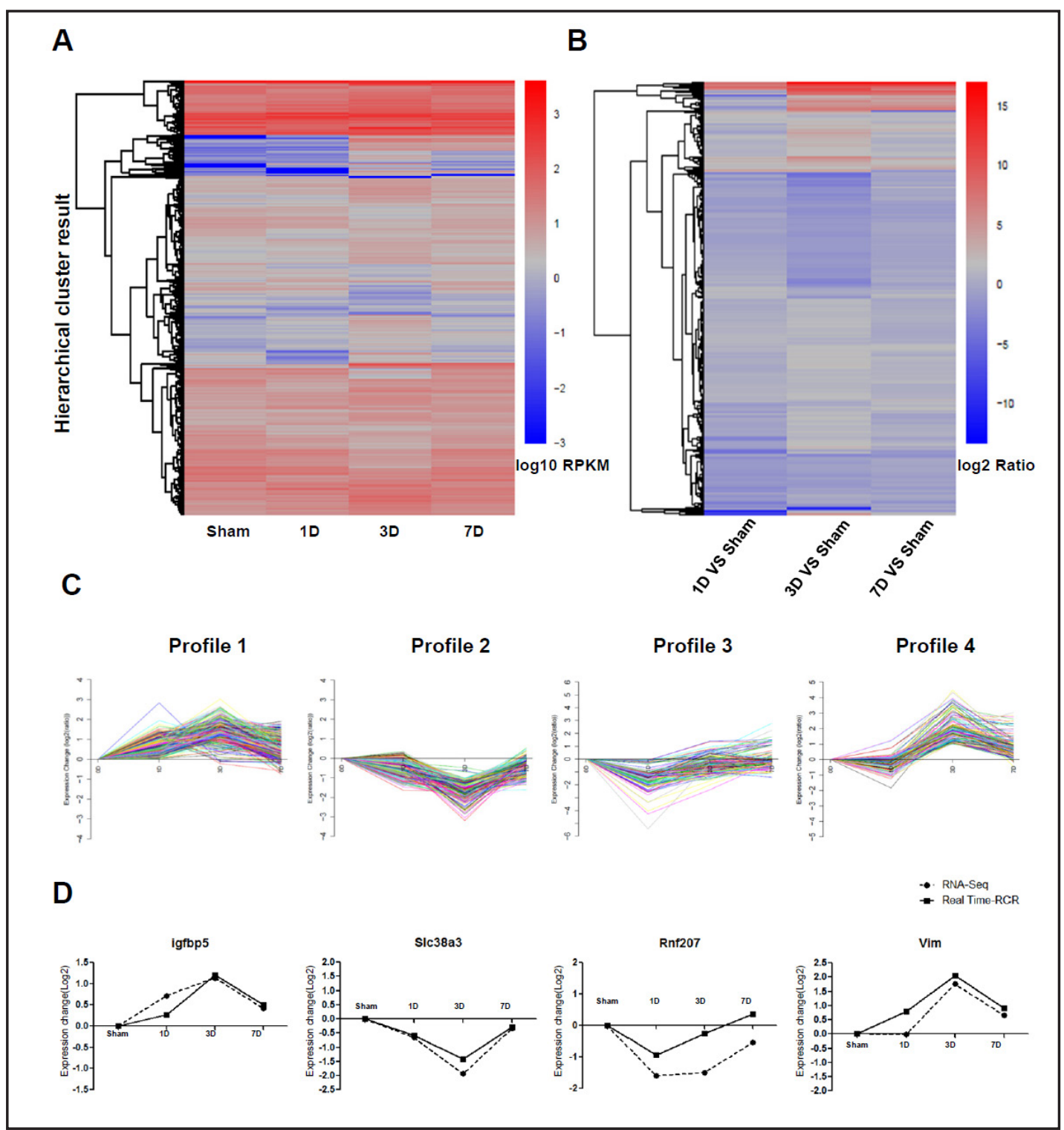

Fig. 5. Cluster analysis of differential genes. (A) For samples' gene expression, log10 (RPKM) is clustered, and the color is more close to red, the more highly this gene expresses. (B) Hierarchical clustering of fold change in expression at day 1, 3, 7 compared with Sham. (C) Gene expression patterns of top 4 profiles containing a total of 1262 genes. (D) Validation of RNA-Seq data by quantitative real-time PCR, including Igfbp5, Slc38a3, Rnf207, Vim.

databases such as BIND, BioGrid and HPRD [28]. The 1,801 genes differentially expressed in heats were analyzed and the correlations between genes were shown in Fig. 6. In the networks, cycle nodes represent genes, and edges between two nodes represent interactions between genes. The results showed ISG15 ubiquitin-like modifier (Isg15) connected with 45 genes, cyclin-dependent kinase 1 (Cdk1) connected with 44 genes at day 1 after Ang II infusion; ubiquitin C connected with 118 genes, ring finger protein 2 (Rnf2) connected with 90 genes at day 3 after Ang II infusion; runt related transcription factor 1(Runx1) connected with 40 genes; v-crk avian sarcoma virus CT10 oncogene homolog (Crk) connected with 23 genes at day 7 after Ang II infusion. Among these genes, ubiquitin C connected with genes mostly, which indicated it may play a vital role in the process of Ang II-induced cardiac inflammation and fibrosis. 


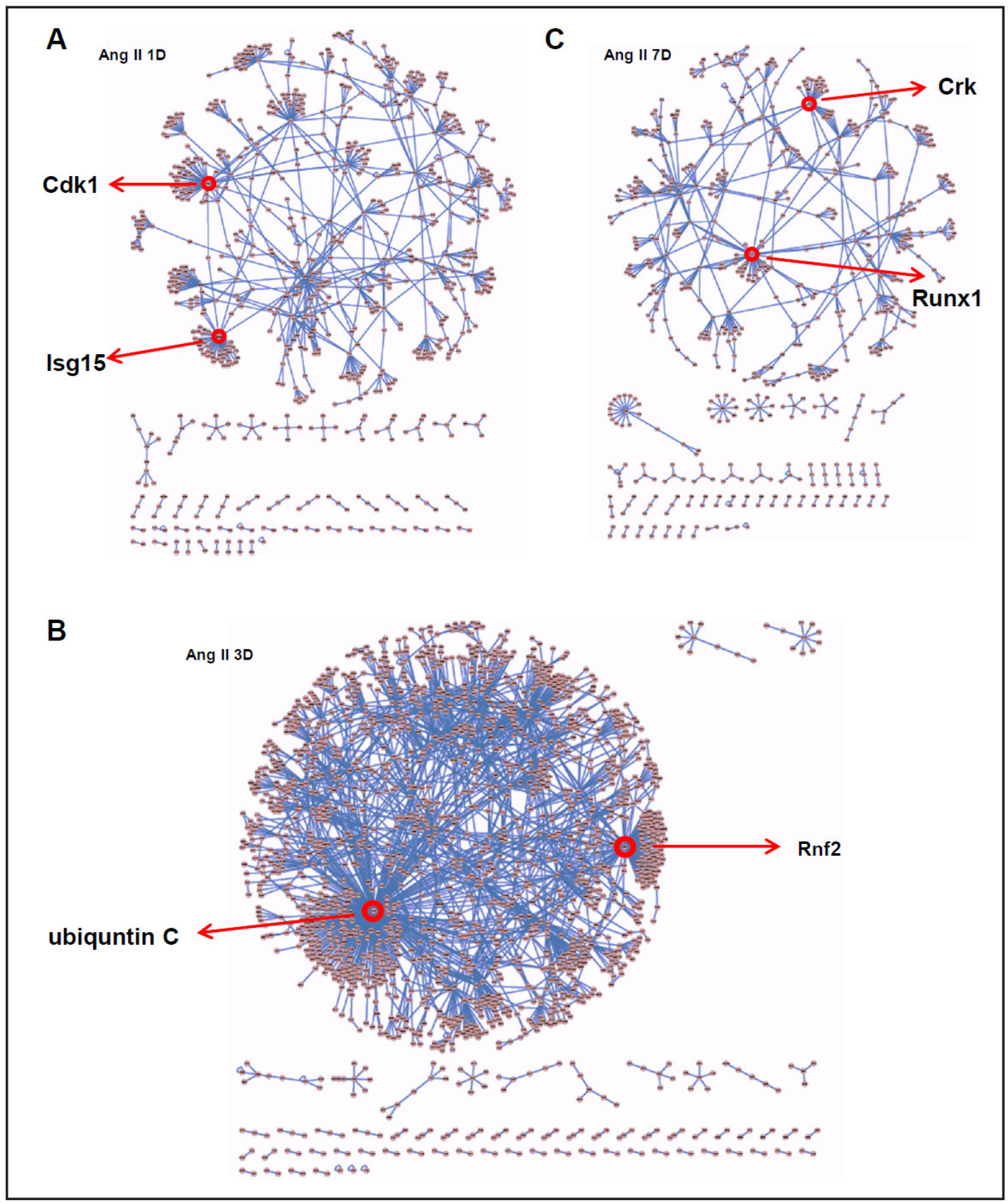

Fig. 6. Protein-protein interaction network analysis. (A) Network analysis for the DEGs at day 1 after Ang II infusion. (B) Network analysis for the DEGs at day 3 after Ang II infusion. (C) Network analysis for the DEGs at day 7 after Ang II infusion. The functional network was created by Cytoscape Software. Cycle nodes represent genes, and edges between two nodes represent interactions between genes. In the network, ubiquitin C connected with 118 genes, and other genes including Isg15, Cdk1, Rnf2, Runx1, Crk interacted with more than 10 genes.

\section{Discussion}

In this study, we investigated the global molecular events associated with the mechanism of hypertension induced cardiac injury using RNA-Seq analysis in a mouse model. Our results showed that totally 1,801 genes were differently expressed at 1, 3 and 7 days after Ang II infusion. Go and KEGG analysis showed that Go terms including response to stress, response to 
wounding, cellular component organization, cell activation, defense response and pathways associated with ECM-receptor interaction, focal adhesion, protein digestion and absorption, phagosome and asthma, played critical roles in the pathobiology of hypertension-induced early cardiac injury. Besides, the gene network analysis demonstrated that ubiquitin $\mathrm{C}$ may play a key role in regulating the processes of hypertension-induced cardiac injury.

Our study showed that neutrophil, macrophage and other leukocyte infiltrations were significantly increased after Ang II infusion (Fig. 1). Moreover, the number of neutrophil infiltrates peaked at day 1 after infusion, and then decreased, while macrophage infiltrated mostly at day 7 after infusion. This phenomenon could be explained as following. As a major blood leukocyte population, neutrophil functions as first responder at sites of infection or injury $[29,30]$. Neutrophils have a rapid rate of turnover. After being recruited to the site of injury, they exert their function for $1-2$ days before undergoing apoptosis [31]. Subsequently, these apoptotic cells and debris were recognized and phagocytized by phagocytic cells such as macrophages [31]. Besides, macrophages directly secreted the cytokines and growth factors activating fibroblasts and other cells. Activation of fibroblasts to myofibroblasts, which can express $\alpha$-smooth muscle actin ( $\alpha$-SMA) and produce ECM components, is a key event in connective tissue remodeling $[32,33]$. Consistent with this, the Go analysis showed that the high enrichment GO terms were mainly response to stress, immune system process, leucocyte migration at day 1 , while the terms were mainly cellular component organization, extracellular structure organization and extracellular matrix organization at day 7 after Ang II infusion.

The transcriptome is the complete set of gene expressions in a cell for a specific developmental stage or physiological condition. The ability to analyze the transcriptome is essential for interpreting the functional elements of the genome, revealing the molecular constituents of cells and tissues, and also for understanding development and disease [12]. Various technologies have been developed to deduce and quantify the transcriptome, including microarrays or RNA-Seq [34]. To date, several studies comparing microarrays and RNA-Seq have been performed [11, 35, 36]. The comparison data derived from microarrays and RNA-Seq using the same set of samples showed a correlation between gene expression profiles generated by the two platforms. However, it also demonstrated that RNA-Seq was superior to microarrays. Marioni, et al. found that RNA-Seq data on the Illumina platform was highly reproducible, with relatively little technical variation [35]. Fu et al. found RNASeq provided better estimates of absolute transcript levels. They also showed the accuracy and precision of RNA-Seq in quantifying the gene expression levels [36]. In the study of Dang, the author used microarrays analyzing the gene expression profiles in the heart after Ang II infusion [37]. The microarray results identified 1,489 differentially expressed genes, while our results showed 1,801 genes were differentially expressed after Ang II infusion at 1, 3 and 7 days. The Go and KEGG pathway analysis of our studies all demonstrated immune response, extracellular matrix organization, cytokine-cytokine receptor interaction, ECM-receptor interaction et al. participate in the development of cardiac injury. By using clustering and gene co-expression network analysis, Dang found that Serpine1 (also known as plasminogen activator inhibitor type 1, PAI-1) was localized in core of the gene network and may play a central role in the progression of Ang II-induced cardiac remodeling. We found ubiquitin C may be the important gene in this pathology by using protein-protein interaction network analysis integrating several famous interaction network databases such as BIND, BioGrid and HPRD [28].

The protein-protein network analysis in Fig. 6 showed ubiquitin C connected with 118 genes, and may be the important gene in regulating the progression of hypertensioninduced cardiac remodeling. Ubiquitin $\mathrm{C}$ represents an ubiquitin gene. The encoded protein is a poly ubiquitin precursor. Conjugation of ubiquitin monomers or polymers can lead to various effects within a cell, depending on the residues to which ubiquitin is conjugated [38]. Phosphorylation, post-translational modification of proteins with ubiquitin can result in the regulation of numerous cellular functions, such as the DNA damage response, apoptosis, cell growth, and the innate immune response. Recently, studies have described

\section{KARGER}




\section{Cellular Physiology Cell Physiol Biochem 2016;38:670-682 \begin{tabular}{ll|l} 
DOI: 10.1159/000438659 & (0) 2016 The Author(s). Published by S. Karger AG, Basel \\
www.karger.com/cpb
\end{tabular} \\ Li et al.: Transcriptome Analysis in Cardic Injury}

how ubiquitination in the heart regulates key signaling transduction pathways important in common cardiac diseases, including cardiac hypertrophy, heart failure, ischemia reperfusion injury, and diabetes [39]. For example, it is reported that the ubiquitin ligase could regulate protein levels of p53 by ubiquitinating p53 [40,41]. Elevated levels of cardiac p53 are associated with a number of cardiovascular conditions including heart failure; cardiac hypertrophy induced by pressure overload and dilated cardiomyopathy (DCM) [42-44]. In the case of DCM, a role for ubiquitin-mediated regulation of p53 has also been found [44]. The KEGG pathway analysis in our study showed the development of cardiac pathology could also be mediated by p53 pathway (Fig. 4). There is growing evidence that ubiquitination plays a role in regulating MAPK signaling in general, and JNK signaling in particular [45]. In the context of cardiac disease, JNK signaling is activated in response to ischemia reperfusion (I/R) injury [46, 47]. An upregulation of cardiac FoxO and p27 expression has been reported to be associated with myocardial infarction, myocardial reperfusion injury, heart failure and myocyte hypertrophy [48-51]. Like the regulation of JNK signaling, recent studies have demonstrated that the heart also contains ubiquitin ligases capable of regulating FoxO signaling [52]. Except for ubiquitin C, the network analysis showed other genes, including Isg15, Cdk1, Rnf2, Runx1and Crk interacted with more than 10 genes, which indicating they may also play a vital role in regulating the pathology of hypertensive heart injury.

In this study, we investigated the global molecular events associated with the mechanism of hypertension induced cardiac injury using RNA-Seq analysis. And our study provides a comprehensive understanding of the transcriptome events in the cardiac pathology. We found that 1,801 genes were differently expressed in the hearts at 1, 3 and 7 days after Ang II infusion. Through a protein-protein interaction network analysis, we identified ubiquitin C may be the important gene in regulating hypertension-induced cardiac injury. However, the exact role of ubiquitin $\mathrm{C}$ in this process remains to be investigated.

\section{Acknowledgements}

We gratefully thank Wei Cui for her excellent technical assistance. The present study was supported by National Natural Science Foundation of China (81230006, 81100094, 81430050, 91339000 and 81100144).

\section{Disclosure Statement}

None.

\section{References}

1 McCormack T, Krause T, O'Flynn N: Management of hypertension in adults in primary care: Nice guideline. Br J Gen Pract 2012;62:163-164.

2 Susic D, Frohlich ED: Hypertensive cardiovascular and renal disease and target organ damage: Lessons from animal models. Cardiorenal Med 2011;1:139-146.

3 Yang M, Zheng J, Miao Y, Wang Y, Cui W, Guo J, Qiu S, Han Y, Jia L, Li H, Cheng J, Du J: Serum-glucocorticoid regulated kinase 1 regulates alternatively activated macrophage polarization contributing to angiotensin ii-induced inflammation and cardiac fibrosis. Arterioscler Thromb Vasc Biol 2012;32:1675-1686.

4 Franco M, Martinez F, Rodriguez-Iturbe B, Johnson RJ, Santamaria J, Montoya A, Nepomuceno T, Bautista R, Tapia E, Herrera-Acosta J: Angiotensin ii, interstitial inflammation, and the pathogenesis of salt-sensitive hypertension. Am J Physiol Renal Physiol 2006;291:F1281-1287.

5 Liu L, Wang C, Sun D, Jiang S, Li H, Zhang W, Zhao Y, Xi Y, Shi S, Lu F, Tian Y, Xu C, Wang L: Calhex(2)(3)(1) ameliorates cardiac hypertrophy by inhibiting cellular autophagy in vivo and in vitro. Cell Physiol Biochem 2015;36:1597-1612. 


\section{Cellular Physiology Cell Physiol Biochem 2016;38:670-682

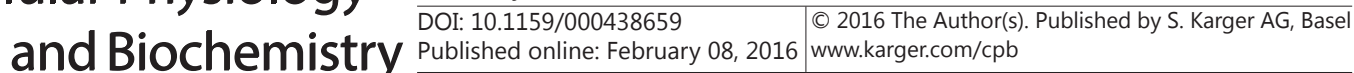 \\ Li et al.: Transcriptome Analysis in Cardic Injury}

6 Jiang G, Gong H, Niu Y, Yang C, Wang S, Chen Z, Ye Y, Zhou N, Zhang G, Ge J, Zou Y: Identification of amino acid residues in angiotensin ii type 1 receptor sensing mechanical stretch and function in cardiomyocyte hypertrophy. Cell Physiol Biochem 2015;37:105-116.

7 Kvakan H, Kleinewietfeld M, Qadri F, Park JK, Fischer R, Schwarz I, Rahn HP, Plehm R, Wellner M, Elitok S, Gratze P, Dechend R, Luft FC, Muller DN: Regulatory t cells ameliorate angiotensin ii-induced cardiac damage. Circulation 2009;119:2904-2912.

8 Liu J, Yang F, Yang XP, Jankowski M, Pagano PJ: Nad(p)h oxidase mediates angiotensin ii-induced vascular macrophage infiltration and medial hypertrophy. Arterioscler Thromb Vasc Biol 2003;23:776-782.

9 Wu Y, Li Y, Zhang C, A X, Wang Y, Cui W, Li H, Du J: S100a8/a9 released by cd11b+gr1+ neutrophils activates cardiac fibroblasts to initiate angiotensin ii-induced cardiac inflammation and injury. Hypertension 2014;63:1241-1250.

10 Kim KH, Moon M, Yu SB, Mook-Jung I, Kim JI: Rna-seq analysis of frontal cortex and cerebellum from 5xfad mice at early stage of disease pathology. J Alzheimers Dis 2012;29:793-808.

11 Zhao S, Fung-Leung WP, Bittner A, Ngo K, Liu X: Comparison of rna-seq and microarray in transcriptome profiling of activated t cells. PLoS One 2014;9:e78644.

12 Wang Z, Gerstein M, Snyder M: Rna-seq: A revolutionary tool for transcriptomics. Nat Rev Genet 2009;10:57-63.

13 Garber M, Grabherr MG, Guttman M, Trapnell C: Computational methods for transcriptome annotation and quantification using rna-seq. Nat Methods 2011;8:469-477.

14 Mortazavi A, Williams BA, McCue K, Schaeffer L, Wold B: Mapping and quantifying mammalian transcriptomes by rna-seq. Nat Methods 2008;5:621-628.

15 Nagalakshmi U, Wang Z, Waern K, Shou C, Raha D, Gerstein M, Snyder M: The transcriptional landscape of the yeast genome defined by rna sequencing. Science 2008;320:1344-1349.

16 Cloonan N, Forrest AR, Kolle G, Gardiner BB, Faulkner GJ, Brown MK, Taylor DF, Steptoe AL, Wani S, Bethel G, Robertson AJ, Perkins AC, Bruce SJ, Lee CC, Ranade SS, Peckham HE, Manning JM, McKernan KJ, Grimmond SM: Stem cell transcriptome profiling via massive-scale mrna sequencing. Nat Methods 2008;5:613-619.

17 Lee JH, Gao C, Peng G, Greer C, Ren S, Wang Y, Xiao X: Analysis of transcriptome complexity through rna sequencing in normal and failing murine hearts. Circ Res 2011;109:1332-1341.

18 Zhang Y, Matkovich SJ, Duan X, Diwan A, Kang MY, Dorn GW, 2nd: Receptor-independent protein kinase c alpha (pkcalpha) signaling by calpain-generated free catalytic domains induces hdac5 nuclear export and regulates cardiac transcription. J Biol Chem 2011;286:26943-26951.

19 Zhang Y, Matkovich SJ, Duan X, Gold JI, Koch WJ, Dorn GW, 2nd: Nuclear effects of g-protein receptor kinase 5 on histone deacetylase 5-regulated gene transcription in heart failure. Circ Heart Fail 2011;4:659-668.

20 Papait R, Cattaneo P, Kunderfranco P, Greco C, Carullo P, Guffanti A, Vigano V, Stirparo GG, Latronico MV, Hasenfuss G, Chen J, Condorelli G: Genome-wide analysis of histone marks identifying an epigenetic signature of promoters and enhancers underlying cardiac hypertrophy. Proc Natl Acad Sci U S A 2013;110:20164-20169.

21 Wang L, Li YL, Zhang CC, Cui W, Wang X, Xia Y, Du J, Li HH: Inhibition of toll-like receptor 2 reduces cardiac fibrosis by attenuating macrophage-mediated inflammation. Cardiovasc Res 2014;101:383-392.

22 Zhang C, Li Y, Wang C, Wu Y, Cui W, Miwa T, Sato S, Li H, Song WC, Du J: Complement 5a receptor mediates angiotensin ii-induced cardiac inflammation and remodeling. Arterioscler Thromb Vasc Biol 2014;34:1240-1248.

23 Li R, Yu C, Li Y, Lam TW, Yiu SM, Kristiansen K, Wang J: Soap2: An improved ultrafast tool for short read alignment. Bioinformatics 2009;25:1966-1967.

24 Kim KI, van de Wiel MA: Effects of dependence in high-dimensional multiple testing problems. BMC Bioinformatics 2008;9:114.

25 Kanehisa M, Araki M, Goto S, Hattori M, Hirakawa M, Itoh M, Katayama T, Kawashima S, Okuda S, Tokimatsu T, Yamanishi Y: Kegg for linking genomes to life and the environment. Nucleic Acids Res 2008;36:D480-484.

26 de Hoon MJ, Imoto S, Nolan J, Miyano S: Open source clustering software. Bioinformatics 2004;20:14531454.

27 Saldanha AJ: Java treeview--extensible visualization of microarray data. Bioinformatics 2004;20:32463248.

28 Hooper SD, Bork P: Medusa: A simple tool for interaction graph analysis. Bioinformatics 2005;21:44324433. 


\section{Cellular Physiology Cell Physiol Biochem 2016;38:670-682

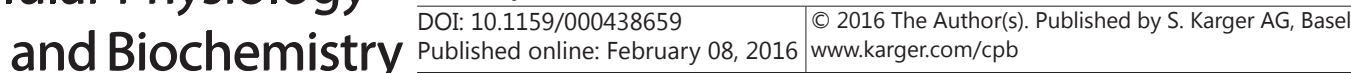 \\ Li et al.: Transcriptome Analysis in Cardic Injury}

29 Savill J, Dransfield I, Gregory C, Haslett C: A blast from the past: Clearance of apoptotic cells regulates immune responses. Nat Rev Immunol 2002;2:965-975.

30 Filep JG, El Kebir D: Neutrophil apoptosis: A target for enhancing the resolution of inflammation. J Cell Biochem 2009;108:1039-1046.

31 Fox S, Leitch AE, Duffin R, Haslett C, Rossi AG: Neutrophil apoptosis: Relevance to the innate immune response and inflammatory disease. J Innate Immun 2010;2:216-227.

32 Jia L, Li Y, Xiao C, Du J: Angiotensin ii induces inflammation leading to cardiac remodeling. Front Biosci (Landmark Ed) 2012;17:221-231.

33 Qi HP, Wang Y, Zhang QH, Guo J, Li L, Cao YG, Li SZ, Li XL, Shi MM, Xu W, Li BY, Sun HL: Activation of peroxisome proliferator-activated receptor gamma (ppargamma) through nf-kappab/brg1 and tgfbeta1 pathways attenuates cardiac remodeling in pressure-overloaded rat hearts. Cell Physiol Biochem 2015;35:899-912.

34 Marguerat S, Bahler J: Rna-seq: From technology to biology. Cell Mol Life Sci 2010;67:569-579.

35 Marioni JC, Mason CE, Mane SM, Stephens M, Gilad Y: Rna-seq: An assessment of technical reproducibility and comparison with gene expression arrays. Genome Res 2008;18:1509-1517.

36 Fu X, Fu N, Guo S, Yan Z, Xu Y, Hu H, Menzel C, Chen W, Li Y, Zeng R, Khaitovich P: Estimating accuracy of rna-seq and microarrays with proteomics. BMC Genomics 2009;10:161.

37 Dang MQ, Zhao XC, Lai S, Wang X, Wang L, Zhang YL, Liu Y, Yu XH, Li HH, Xia YL: Gene expression profile in the early stage of angiotensin ii-induced cardiac remodeling: A time series microarray study in a mouse model. Cell Physiol Biochem 2015;35:467-476.

38 Fang Y, Fu D, Shen XZ: The potential role of ubiquitin c-terminal hydrolases in oncogenesis. Biochim Biophys Acta 2010;1806:1-6.

39 Portbury AL, Ronnebaum SM, Zungu M, Patterson C, Willis MS: Back to your heart: Ubiquitin proteasome system-regulated signal transduction. J Mol Cell Cardiol 2012;52:526-537.

40 Dolezelova P, Cetkovska K, Vousden KH, Uldrijan S: Mutational analysis reveals a dual role of mdm2 acidic domain in the regulation of p53 stability. FEBS Lett 2012;586:2225-2231.

41 Qiu W, Wu J, Walsh EM, Zhang Y, Chen CY, Fujita J, Xiao ZX: Retinoblastoma protein modulates gankyrin$\mathrm{mdm} 2$ in regulation of p53 stability and chemosensitivity in cancer cells. Oncogene 2008;27:4034-4043.

42 Sano M, Minamino T, Toko H, Miyauchi H, Orimo M, Qin Y, Akazawa H, Tateno K, Kayama Y, Harada M, Shimizu I, Asahara T, Hamada H, Tomita S, Molkentin JD, Zou Y, Komuro I: P53-induced inhibition of hif-1 causes cardiac dysfunction during pressure overload. Nature 2007;446:444-448.

43 Chatterjee A, Mir SA, Dutta D, Mitra A, Pathak K, Sarkar S: Analysis of p53 and nf-kappab signaling in modulating the cardiomyocyte fate during hypertrophy. J Cell Physiol 2011;226:2543-2554.

44 Birks EJ, Latif N, Enesa K, Folkvang T, Luong le A, Sarathchandra P, Khan M, Ovaa H, Terracciano CM, Barton PJ, Yacoub MH, Evans PC: Elevated p53 expression is associated with dysregulation of the ubiquitinproteasome system in dilated cardiomyopathy. Cardiovasc Res 2008;79:472-480.

45 Laine A, Ronai Z: Ubiquitin chains in the ladder of mapk signaling. Sci STKE 2005;2005:re5.

46 Sugden PH, Clerk A: "Stress-responsive" mitogen-activated protein kinases (c-jun n-terminal kinases and p38 mitogen-activated protein kinases) in the myocardium. Circ Res 1998;83:345-352.

47 Yin T, Sandhu G, Wolfgang CD, Burrier A, Webb RL, Rigel DF, Hai T, Whelan J: Tissue-specific pattern of stress kinase activation in ischemic/reperfused heart and kidney. J Biol Chem 1997;272:19943-19950.

48 Hannenhalli S, Putt ME, Gilmore JM, Wang J, Parmacek MS, Epstein JA, Morrisey EE, Margulies KB, Cappola TP: Transcriptional genomics associates fox transcription factors with human heart failure. Circulation 2006;114:1269-1276.

49 Sengupta A, Molkentin JD, Paik JH, DePinho RA, Yutzey KE: Foxo transcription factors promote cardiomyocyte survival upon induction of oxidative stress. J Biol Chem 2011;286:7468-7478.

50 Yue TL, Bao W, Gu JL, Cui J, Tao L, Ma XL, Ohlstein EH, Jucker BM: Rosiglitazone treatment in zucker diabetic fatty rats is associated with ameliorated cardiac insulin resistance and protection from ischemia/ reperfusion-induced myocardial injury. Diabetes 2005;54:554-562.

51 Liu Y, Zhou D, Li G, Ming X, Tu Y, Tian J, Lu H, Yu B: Long non coding rna-uca1 contributes to cardiomyocyte apoptosis by suppression of p27 expression. Cell Physiol Biochem 2015;35:1986-1998.

52 Li HH, Willis MS, Lockyer P, Miller N, McDonough H, Glass DJ, Patterson C: Atrogin-1 inhibits akt-dependent cardiac hypertrophy in mice via ubiquitin-dependent coactivation of forkhead proteins. J Clin Invest 2007;117:3211-3223. 